\title{
Supernova Neutrino Oscillations and the Neutrino Magnetic Moment
}

\author{
Shin'ichiro Ando* and Katsuhiko Sato ${ }^{\dagger}$ \\ Department of Physics, School of Science, The University of Tokyo, 7-3-1 Hongo, \\ Bunkyo-ku, Tokyo 113-0033, Japan \\ E-mail: ando@utap. phys.s.u-tokyo ac jpi, satoophys s.u-tokyo.ac.jp
}

\begin{abstract}
Resonant spin-flavour (RSF) conversions of supernova neutrinos, which are induced by the interaction between the nonzero neutrino magnetic moment and supernova magnetic fields, are studied for both normal and inverted mass hierarchy. As the case for the pure matter-induced neutrino oscillation (Mikheyev-Smirnov-Wolfenstein (MSW) effect), we find that the RSF transitions are strongly dependent on the neutrino mass hierarchy as well as the value of $\theta_{13}$. Flavour conversions are solved numerically for various neutrino parameter sets, with the realistic presupernova profile. In particular, it is very interesting that the RSF-induced $\nu_{\mathrm{e}} \rightarrow \bar{\nu}_{\mathrm{e}}$ transition occurs if the following conditions are all satisfied: the value of $\mu_{\nu} B$ ( $\mu_{\nu}$ is the neutrino magnetic moment and $B$ is the magnetic field strength) is sufficiently strong, the neutrino mass hierarchy is inverted, and the value of $\theta_{13}$ is large enough to induce adiabatic MSW resonance. In this case, the strong peak due to the original $\nu_{\mathrm{e}}$ emitted from the neutronization burst would exist in the time profile of the neutrino events detected at the Super-Kamiokande detector. If this peak were observed in reality, it would provide fruitful information on the neutrino properties. On the other hand, the characteristics of the neutrino spectra are also different between the neutrino models, but we find that there remains degeneracy among several models. This contribution is based on our recent study [i1,
\end{abstract}

KEYwORDS: 'supernovas, neutrino properties, supernova neutrinosi.

\section{Introduction}

A core-collapse supernova explosion is one of the most spectacular events in astrophysics, and it attracts a great deal of attention from many physicists and astronomers. It also

\footnotetext{
${ }^{*}$ Speaker.

${ }^{\dagger}$ Research Center for the Early Universe, School of Science, The University of Tokyo, 7-3-1 Hongo, Bunkyo-ku, Tokyo 113-0033, Japan
} 
produces a number of neutrinos and $99 \%$ of its gravitational binding energy is transformed to neutrinos. Therefore, neutrinos play an essential role in supernovae, and their detection by ground-based large water Čerenkov detectors, such as Super-Kamiokande (SK) and Sudbury Neutrino Observatory (SNO), would provide valuable information on the nature of neutrinos as well as supernova physics. What we can learn from the next galactic supernova has been considered in many articles (for a review, see [2i2i). For example, we can constrain the properties of neutrino oscillations, such as the mixing angle between the first and third mass eigenstates $\left(\theta_{13}\right)$, and the mass hierarchy (normal, $m_{1} \ll m_{3}$, or inverted, $\left.m_{1} \gg m_{3}\right)[\overline{3} \overline{3}]$.

In addition to the nonzero neutrino masses and mixing angles, the nonzero magnetic moment is another property of neutrinos beyond the standard model of particle physics. If neutrinos have a nonzero magnetic moment, it leads to precession between left- and right-handed neutrinos in sufficiently strong magnetic fields [i $\left[\begin{array}{l}\overline{4} \\ ]\end{array}\right]$. In general, nondiagonal elements of the magnetic moment matrix are possible and neutrinos can be changed into different flavours and chiralities [i5i]. Furthermore, with the additional effect of coherent forward scattering by matter, neutrinos can be resonantly converted into ones with different

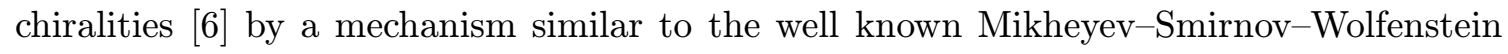
(MSW) effect [i]. This resonant spin-flavour (RSF) conversion induced by the neutrino magnetic moment in strong magnetic fields was first introduced to solve the solar neutrino problem, and actually gave the best fit solution before the KamLAND result [i 87. . However, the recent KamLAND experiment [9i] has shown that the large mixing angle (LMA) MSW solution is the most favourable one; the RSF mechanism is suppressed at the subdominant level. From the KamLAND negative results for the solar antineutrino search, an upper bound on the neutrino magnetic moment is obtained, $\mu_{\nu} \lesssim 1 \times 10^{-12} \mu_{\mathrm{B}}$, where $\mu_{\mathrm{B}}$ is the Bohr magneton [1] $\left.0_{1}^{1}\right]$. This upper bound is comparable to the most stringent limit from the stellar cooling argument, $\mu_{\nu} \lesssim(1-4) \times 10^{-12} \mu_{\mathrm{B}}\left[\underline{1} \mathbf{1}_{1} \overline{1}_{1}\right.$.

Although the RSF mechanism does not work at a dominant level in the Sun, it may occur efficiently in a denser environment with stronger magnetic field, which is actually expected in the case of core-collapse supernovae. The RSF conversion mechanism in su-

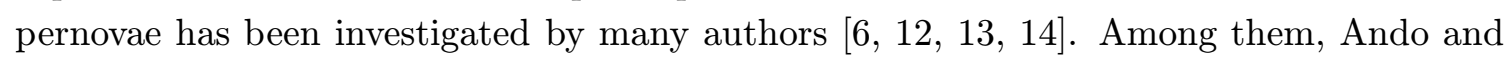
Sato [13 3 in have studied the RSF effect using a three-flavour formulation with the latest oscillation parameters, and pointed out that the combination of the MSW and RSF effects makes the crossing scheme very interesting to investigate. Since the RSF conversions are very sensitive to the value of $Y_{\mathrm{e}}$, which is the electron number fraction per nucleon, they have also investigated the dependence of the RSF effect on presupernova models with solar and zero metallicities [1 14 in]. It is concluded that the efficient (either complete or incomplete, depending on the presupernova models) RSF conversions occur when the supernova magnetic field is sufficiently strong, $\mu_{\nu} B_{0} \simeq\left(10^{-12} \mu_{\mathrm{B}}\right)\left(10^{10} \mathrm{G}\right)$, where $B_{0}$ is the strength of the magnetic field at the surface of the iron core.

However, all the past studies of the three-flavour RSF effect were based on the assumption that the neutrino mass hierarchy is normal $\left(m_{1} \ll m_{3}\right)$, although the inverted mass hierarchy $\left(m_{1} \gg m_{3}\right)$ has not been excluded at all. For the pure MSW effect, it is well known that the supernova neutrino signal with the case of inverted hierarchy would be very 
different from that with normal hierarchy [3]. From the analogy of the conversion mechanisms between the MSW and RSF effects, it is easily inferred that the RSF conversions will also be very sensitive to the mass hierarchy. Therefore, in this contribution, we study three-flavour RSF conversions with the inverted mass hierarchy using the latest neutrino mixing parameters, and give a comprehensive discussion concerning the dependence on the mass hierarchy. In particular, we show that the RSF conversion in the case of the inverted hierarchy with the large $\theta_{13}$ causes a very different neutrino signal from the other models, i.e., the appearance of a sharp peak of the neutronization burst in the $\bar{\nu}_{\mathrm{e}}$ time profile. If this case were actually realized, it would be clearly confirmed by not only the neutrino spectrum but also the luminosity curve.

\section{Formulation}

\subsection{Interaction with matter and magnetic fields}

The interaction of the magnetic moment of neutrinos and magnetic fields is described by

$$
\left\langle\left(\nu_{\mathrm{i}}\right)_{\mathrm{R}}\left|H_{\mathrm{int}}\right|\left(\nu_{\mathrm{j}}\right)_{\mathrm{L}}\right\rangle=\mu_{\mathrm{ij}} B_{\perp},
$$

where $\mu_{\mathrm{ij}}$ is the component of the neutrino magnetic moment matrix, $B_{\perp}$ is the magnetic field transverse to the direction of propagation, and $(\nu)_{\mathrm{R}}$ and $(\nu)_{\mathrm{L}}$ are the right- and lefthanded neutrinos, respectively. If neutrinos are Dirac particles, right-handed neutrinos and left-handed antineutrinos are undetectable (sterile neutrinos), since they do not interact with matter. On the other hand, if neutrinos are Majorana particles, $\nu_{\mathrm{R}}$ are identical to antiparticles of $\nu_{\mathrm{L}}$ and interact with matter. In this paper, we assume that neutrinos are Majorana particles. The diagonal magnetic moments are forbidden for Majorana neutrinos, and therefore only conversion between different flavours is possible, e.g., $\left(\bar{\nu}_{\mathrm{e}}\right)_{\mathrm{R}} \leftrightarrow\left(\nu_{\mu, \tau}\right)_{\mathrm{L}}$ or $\left(\nu_{\mathrm{e}}\right)_{\mathrm{L}} \leftrightarrow\left(\bar{\nu}_{\mu, \tau}\right)_{\mathrm{R}}$.

Coherent forward scattering with matter induces an effective potential for neutrinos, which is calculated using weak interaction theory. The effective potential due to scattering with electrons is given by

$$
V_{ \pm \pm}= \pm \sqrt{2} G_{\mathrm{F}}\left( \pm \frac{1}{2}+2 \sin ^{2} \theta_{\mathrm{W}}\right) n_{\mathrm{e}}
$$

where $n_{\mathrm{e}}$ is the electron number density, $G_{\mathrm{F}}$ is the Fermi coupling constant, and $\theta_{\mathrm{W}}$ is the Weinberg angle. The first \pm sign in front refers to $\nu(+)$ and $\bar{\nu}(-)$ and that in the parentheses to $\nu_{\mathrm{e}}(+)$ and $\nu_{\mu, \tau}(-)$. The difference between e and $\mu, \tau$ neutrinos comes from the existence of charged-current interaction. The subscript \pm \pm of $V$ refers to the first and the second \pm sign. The ordinary MSW effect between $\nu_{\mathrm{e}}$ and $\nu_{\mu, \tau}$ is caused by the potential difference $V_{\mathrm{e}}-V_{\mu, \tau}=V_{++}-V_{+-}=\sqrt{2} G_{\mathrm{F}} n_{\mathrm{e}}$, while that between $\bar{\nu}_{\mathrm{e}}$ and $\bar{\nu}_{\mu, \tau}$ by $V_{\overline{\mathrm{e}}}-V_{\bar{\mu}, \bar{\tau}}=V_{-+}-V_{--}=-\sqrt{2} G_{\mathrm{F}} n_{\mathrm{e}}$. To include the RSF effect, which causes conversion between neutrinos and antineutrinos, we should take into account the neutralcurrent scattering by nucleons:

$$
V=\sqrt{2} G_{\mathrm{F}}\left(\frac{1}{2}-2 \sin ^{2} \theta_{\mathrm{W}}\right) n_{\mathrm{p}}-\sqrt{2} G_{\mathrm{F}} \frac{1}{2} n_{\mathrm{n}}
$$


where $n_{\mathrm{p}}, n_{\mathrm{n}}$ are the proton and neutron number density, respectively. For neutrinos we add $+V$ to the potential and for antineutrinos $-V$. Therefore, the RSF conversion between $\nu_{\mathrm{e}}$ and $\bar{\nu}_{\mu, \tau}$, which is important for the case considered in this paper, obeys the potential difference

$$
\Delta V \equiv V_{\mathrm{e}}-V_{\bar{\mu}, \bar{\tau}}=\left(V_{++}+V\right)-\left(V_{--}-V\right)=-\sqrt{2} G_{\mathrm{F}} \frac{\rho}{m_{\mathrm{N}}}\left(1-2 Y_{\mathrm{e}}\right)
$$

where $\rho$ is the density, $m_{\mathrm{N}}$ is the nucleon mass, and $Y_{\mathrm{e}}=n_{\mathrm{e}} /\left(n_{\mathrm{e}}+n_{\mathrm{n}}\right)$ is the number of electrons per baryon. (When we obtained equation $(\overline{2} . \overline{4})$, we assumed charge neutrality, $\left.n_{\mathrm{e}}=n_{\mathrm{p}} \cdot\right)$

\subsection{Three-flavour formulation}

Here, we present the three-flavour (six-component) formulation of neutrino mixing, on which our discussions depend:

$$
\mathrm{i} \frac{\mathrm{d}}{\mathrm{d} r}\left(\begin{array}{c}
\nu \\
\bar{\nu}
\end{array}\right)=\left(\begin{array}{cc}
H_{0} & B_{\perp} M \\
-B_{\perp} M & \bar{H}_{0}
\end{array}\right)\left(\begin{array}{c}
\nu \\
\bar{\nu}
\end{array}\right),
$$

where

$$
\begin{aligned}
& \nu=\left(\begin{array}{c}
\nu_{\mathrm{e}} \\
\nu_{\mu} \\
\nu_{\tau}
\end{array}\right), \bar{\nu}=\left(\begin{array}{c}
\bar{\nu}_{\mathrm{e}} \\
\bar{\nu}_{\mu} \\
\bar{\nu}_{\tau}
\end{array}\right) \\
& H_{0}=\frac{1}{2 E_{\nu}} U\left(\begin{array}{ccc}
0 & 0 & 0 \\
0 & \Delta m_{12}^{2} & 0 \\
0 & 0 & \Delta m_{13}^{2}
\end{array}\right) U^{\dagger}+\left(\begin{array}{ccc}
V_{++}+V & 0 & 0 \\
0 & V_{+-}+V & 0 \\
0 & 0 & V_{+-}+V
\end{array}\right) \text {, } \\
& \bar{H}_{0}=\frac{1}{2 E_{\nu}} U\left(\begin{array}{ccc}
0 & 0 & 0 \\
0 & \Delta m_{12}^{2} & 0 \\
0 & 0 & \Delta m_{13}^{2}
\end{array}\right) U^{\dagger}+\left(\begin{array}{ccc}
V_{-+}-V & 0 & 0 \\
0 & V_{--}-V & 0 \\
0 & 0 & V_{--}-V
\end{array}\right) \text {, } \\
& U=\left(\begin{array}{ccc}
U_{\mathrm{e} 1} & U_{\mathrm{e} 2} & U_{\mathrm{e} 3} \\
U_{\mu 1} & U_{\mu 2} & U_{\mu 3} \\
U_{\tau 1} & U_{\tau 2} & U_{\tau 3}
\end{array}\right)=\left(\begin{array}{ccc}
c_{12} c_{13} & s_{12} c_{13} & s_{13} \\
-s_{12} c_{23}-c_{12} s_{23} s_{13} & c_{12} c_{23}-s_{12} s_{23} s_{13} & s_{23} c_{13} \\
s_{12} s_{23}-c_{12} c_{23} s_{13} & -c_{12} s_{23}-s_{12} c_{23} s_{13} & c_{23} c_{13}
\end{array}\right) \\
& M=\left(\begin{array}{ccc}
0 & \mu_{\mathrm{e} \mu} & \mu_{\mathrm{e} \tau} \\
-\mu_{\mathrm{e} \mu} & 0 & \mu_{\mu \tau} \\
-\mu_{\mathrm{e} \tau} & -\mu_{\mu \tau} & 0
\end{array}\right)
\end{aligned}
$$

and $c_{\mathrm{ij}}=\cos \theta_{\mathrm{ij}}, s_{\mathrm{ij}}=\sin \theta_{\mathrm{ij}}$. (We assume the CP phase $\delta=0$ in equation (2. $\left.\overline{2}_{\mathbf{i}}\right)$ for simplicity.)

The resonant flavour conversion basically occurs when the two diagonal elements in the matrix in equation (2.5. but they depend on the neutrino mass hierarchy. In the case of the normal mass hierarchy, they are for $\nu_{\mathrm{e}} \leftrightarrow \nu_{\mu}^{\prime}$ (MSW-L), $\nu_{\mathrm{e}} \leftrightarrow \nu_{\tau}^{\prime}$ (MSW-H), $\bar{\nu}_{\mathrm{e}} \leftrightarrow \nu_{\mu}^{\prime}$ (RSF-L) and $\bar{\nu}_{\mathrm{e}} \leftrightarrow \nu_{\tau}^{\prime}$ (RSF$\mathrm{H})$. Here, the quantities such as $\nu_{\mu, \tau}^{\prime}\left(\bar{\nu}_{\mu, \tau}^{\prime}\right)$ represent mass eigenstates in matter which can 
be obtained by linear combination of $\nu_{\mu}\left(\bar{\nu}_{\mu}\right)$ and $\nu_{\tau}\left(\bar{\nu}_{\tau}\right)$. This is because the $\nu_{\mathrm{e}}\left(\bar{\nu}_{\mathrm{e}}\right)$ state coincides with the mass eigenstate in matter owing to the large matter potential, and the other mass eigenstates are obtained by the rotation of the $\nu_{\mu}\left(\bar{\nu}_{\mu}\right)$ and $\nu_{\tau}\left(\bar{\nu}_{\tau}\right)$ basis. Suffixes '-L' and '-H' attached to 'MSW' and 'RSF' indicate whether the densities at the resonance points are lower or higher. On the other hand, in the case of the inverted hierarchy, RSF-H occurs for $\nu_{\mathrm{e}} \leftrightarrow \bar{\nu}_{\tau}^{\prime}$ as well as MSW-H for $\bar{\nu}_{\mathrm{e}} \leftrightarrow \bar{\nu}_{\tau}^{\prime}$, whereas the other two resonances occur for the same conversions. This situation is summarized in table

\begin{tabular}{lll}
\hline Resonance & Normal hierarchy & Inverted hierarchy \\
\hline RSF-H & $\bar{\nu}_{\mathrm{e}} \leftrightarrow \nu_{\tau}^{\prime}$ & $\nu_{\mathrm{e}} \leftrightarrow \bar{\nu}_{\tau}^{\prime}$ \\
RSF-L & $\bar{\nu}_{\mathrm{e}} \leftrightarrow \nu_{\mu}^{\prime}$ & $\bar{\nu}_{\mathrm{e}} \leftrightarrow \nu_{\mu}^{\prime}$ \\
MSW-H & $\nu_{\mathrm{e}} \leftrightarrow \nu_{\tau}^{\prime}$ & $\bar{\nu}_{\mathrm{e}} \leftrightarrow \bar{\nu}_{\tau}^{\prime}$ \\
MSW-L & $\nu_{\mathrm{e}} \leftrightarrow \nu_{\mu}^{\prime}$ & $\nu_{\mathrm{e}} \leftrightarrow \nu_{\mu}^{\prime}$ \\
\hline
\end{tabular}

Table 1: Flavour conversions that are important for each resonance, in cases of both normal and inverted mass hierarchy.

\section{Models}

\subsection{Original neutrino emission}

We adopt, as the original neutrino spectrum as well as the luminosity curve, the result of the numerical simulation by Thompson et al. [1] $\left.\overline{1}_{1}\right]$; we use the model calculated for the $15 M_{\odot}$ progenitor star. Their calculation has particularly focused on shock breakout and followed the dynamical evolution of the cores through collapse until the first $250 \mathrm{~ms}$ after bounce. They have incorporated all the relevant neutrino processes such as neutrinonucleon scatterings with nucleon recoil as well as nucleon bremsstrahlung; these reactions have recently been recognized to give non-negligible contribution to the spectral formation. In figures $\underline{1}_{1}^{1}$ and respectively. In these figures, $\nu_{\mathrm{x}}$ represents non-electron neutrinos and antineutrinos.

The neutrino luminosity curve is quite characteristic among the different flavours. In particular, there is a very sharp peak of $\nu_{\mathrm{e}}$ called the 'neutronization burst', whose duration is typically $\sim 10 \mathrm{~ms}$ and peak luminosity is $\sim 10^{53} \mathrm{erg} \mathrm{s}^{-1}$. This strong peak is illustrated as follows. As a supernova shock moves outward, it dissociates nuclei into free nucleons, which triggers the deleptonization process $\mathrm{e}^{-} \mathrm{p} \rightarrow \nu_{\mathrm{e}} \mathrm{n}$; these $\nu_{\mathrm{e}}$ build up a sea because they are trapped and advected with matter. When the shock crosses the $\nu_{\mathrm{e}}$ neutrinosphere, within which the created $\nu_{\mathrm{e}}$ are trapped, they are abruptly emitted. For the other flavours $\bar{\nu}_{\mathrm{e}}$ and $\nu_{\mathrm{x}}$, there is no such a sudden burst; both luminosities glow rather gradually and they are similar to each other. Usually, for both the pure MSW effect and the RSF effect with normal hierarchy, the most easily detected flavour $\bar{\nu}_{\mathrm{e}}$ is transformed from the original

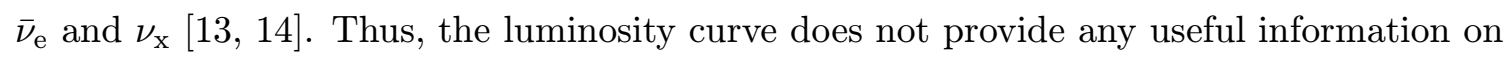
the flavour conversion mechanism. On the other hand, for the RSF effect with inverted mass hierarchy, the conversion $\nu_{\mathrm{e}} \rightarrow \bar{\nu}_{\tau}^{\prime} \rightarrow \bar{\nu}_{\mathrm{e}}$ is considered to occur via RSF-H and MSW-H

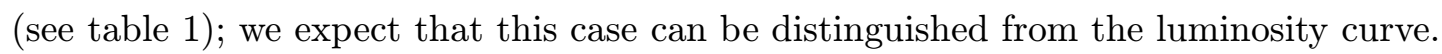




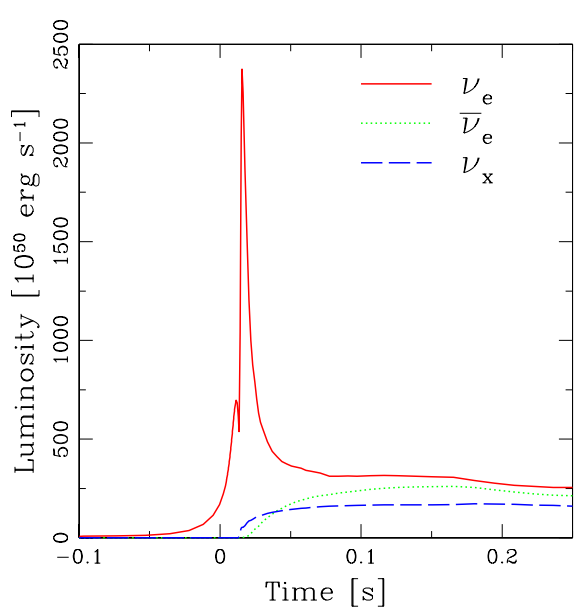

Figure 1: The original luminosity of the emitted neutrinos as a function of time, calculated by Thompson et al. [1 15 in. The progenitor mass is $15 M_{\odot}$.

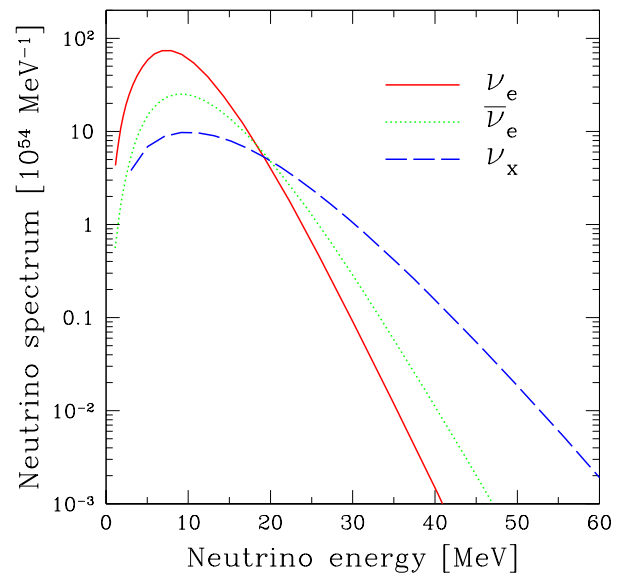

Figure 2: Original neutrino spectrum integrated to $0.25 \mathrm{~s}$ after core bounce, calculated by Thompson et al. [115in. The progenitor mass is $15 M_{\odot}$.

The other characteristic that provides information on the flavour conversion mechanism is the hierarchy of the average energy $\left\langle E_{\nu_{\mathrm{e}}}\right\rangle\left\langle\left\langle E_{\bar{\nu}_{\mathrm{e}}}\right\rangle\left\langle\left\langle E_{\nu_{\mathrm{x}}}\right\rangle\right.\right.$ as clearly seen from figure ${ }^{2} \overline{2}_{;}$ flavour conversions also cause the spectral exchange. This energy hierarchy is explained as follows. Since $\nu_{\mathrm{x}}$ interact with matter only through the neutral-current interactions in supernovae, they are weakly coupled with matter compared to $\nu_{\mathrm{e}}$ and $\bar{\nu}_{\mathrm{e}}$. Thus the neutrinosphere of $\nu_{\mathrm{x}}$ locates deeper in the core than that of $\nu_{\mathrm{e}}$ and $\bar{\nu}_{\mathrm{e}}$, which leads to higher temperatures for $\nu_{\mathrm{x}}$. The difference between $\nu_{\mathrm{e}}$ and $\bar{\nu}_{\mathrm{e}}$ comes from the fact that the core is neutron-rich and the $\nu_{\mathrm{e}}$ couple with matter more strongly, through the $\nu_{\mathrm{e}} \mathrm{n} \rightarrow \mathrm{e}^{-} \mathrm{p}$ reaction.

\subsection{Presupernova profiles}

We use the precollapse model of massive stars of Woosley and Weaver [i] $\left.\overline{6}_{1}^{1}\right]$. The model is the $15 M_{\odot}$ progenitor star with solar metallicity. The density and $Y_{\mathrm{e}}$ profiles are quite important for the flavour conversions because they determine the resonance regions as well as whether it is adiabatic or not. We show in figure $\overline{\beta_{1}}$, the $\left|\rho\left(1-2 Y_{\mathrm{e}}\right)\right|$ (responsible for RSF) and $\rho Y_{\mathrm{e}}$ (responsible for MSW) profiles of this model. We also show $\Delta_{12} \equiv$ $m_{\mathrm{N}} \Delta m_{12}^{2} \cos 2 \theta_{12} / 2 \sqrt{2} G_{\mathrm{F}} E_{\nu}$ and $\Delta_{13} \equiv m_{\mathrm{N}}\left|\Delta m_{13}^{2}\right| \cos 2 \theta_{13} / 2 \sqrt{2} G_{\mathrm{F}} E_{\nu}$ as two horizontal bands (the bandwidth comes from the energy range 5-70 MeV). At intersections between $\Delta_{12}, \Delta_{13}$ and $\left|\rho\left(1-2 Y_{\mathrm{e}}\right)\right|, \rho Y_{\mathrm{e}}$, the RSF and MSW conversions take place.

Although we use static progenitor models in calculating the flavour transition, in fact the density profile changes drastically during a neutrino burst $(\sim 10 \mathrm{~s})$ owing to shock wave propagation. However, our particular interest is within first $0.25 \mathrm{~s}$ after core bounce

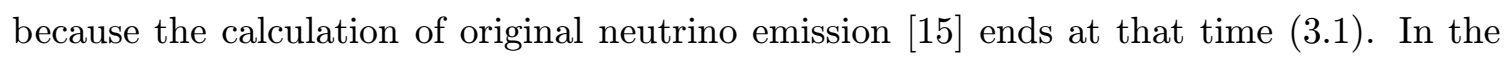
previous paper, we have already shown that until $0.25 \mathrm{~s}$, using the static presupernova and magnetic field models is considered to be a good approximation [i] 1 in]; this is based on the 
numerical calculation of the Lawrence Livermore group, which is the only group succeeding in shock propagation into the outer envelope.

\subsection{Magnetic fields}

We assume that the global structure of the magnetic field is a dipole moment and the field strength is normalized at the surface of the iron core with the values $10^{10} \mathrm{G}$ (nearly complete RSF conversion) as well as $0 \mathrm{G}$ (pure MSW conversion). The strength of such magnetic fields above the surface of the iron core may be inferred from observations of the surface of white dwarfs, since both are sustained against gravitational collapse by the degenerate pressure of electrons. Observations of the magnetic fields in white dwarfs show that the strength spreads in a wide range of $10^{7}-10^{9} \mathrm{G}$ [i] $\overline{1}$ i]. Considering the possibility of the decay of magnetic fields in white dwarfs, it is not unnatural to consider magnetic fields up to $10^{10}$ $\mathrm{G}$ at the surface of the iron core. Then, in

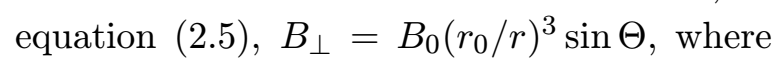
$B_{0}$ is the strength of the magnetic field at the equator on the iron core surface, $r_{0}$ the radius of the iron core, and $\Theta$ the angle between the

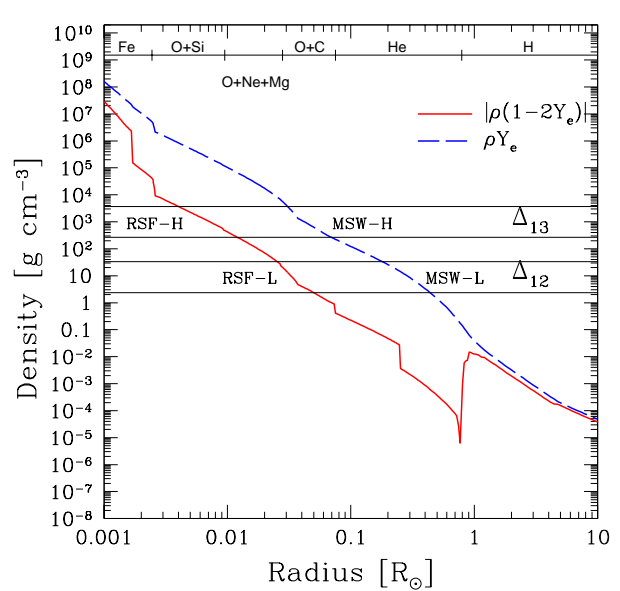

Figure 3: Presupernova profiles used in our calculations, which are calculated by Woosley and Weaver [1 $\left.1 \bar{b}^{-}\right]$. We show the density and $Y_{\mathrm{e}}$ combination that is responsible for the RSF conversions $\left[\rho\left(1-2 Y_{\mathrm{e}}\right)\right]$, and that for the MSW conversions $\left(\rho Y_{\mathrm{e}}\right)$. Two horizontal bands represent $\Delta_{12}$ and $\Delta_{13}$ (these definitions are given in the text); at the intersections between them and the profile curves, the RSF and MSW conversions occur. pole of the magnetic dipole and the direction of neutrino propagation. Hereafter, we assume $\sin \Theta=1$.

\subsection{Neutrino parameters}

We adopt the realistic neutrino mixing parameters inferred from the recent experimental results: for the atmospheric neutrino parameters, $\left|\Delta m_{13}^{2}\right|=2.8 \times 10^{-3} \mathrm{eV}^{2}, \sin ^{2} 2 \theta_{23}=1.0$, and for the solar neutrino parameters, $\Delta m_{12}^{2}=5.0 \times 10^{-5} \mathrm{eV}^{2}, \tan ^{2} \theta_{12}=0.42$.

For still uncertain neutrino properties, we must set some assumptions, e.g., whether the mass hierarchy is normal or inverted, as well as whether $\theta_{13}$ is large enough for the MSW-H conversion to be adiabatic, or not. There is also uncertainty concerning the neutrino magnetic moment tensor $\mu_{\mathrm{ij}}$; however, since the only relevant parameter is $\mu_{\mathrm{ij}} B$, this uncertainty is already included in that of the magnetic field strength $\left(\mu_{\mathrm{ij}}=10^{-12} \mu_{\mathrm{B}}\right.$ is assumed). Thus, there are eight parameter sets due to: whether the magnetic field is zero (labeled by 'MSW') or sufficiently strong $B_{0}=10^{10} \mathrm{G}$ ('RSF'); the mass hierarchy is normal ('NOR') or inverted ('INV'); and $\sin ^{2} 2 \theta_{13}=10^{-6}$ ('S') or $\sin ^{2} 2 \theta_{13}=0.04$ ('L'). We label one model by connecting these labels using hyphens, e.g., MSW-NOR-S. It should be particularly noticed that although we label the model with strong magnetic field simply by RSF-, it does not mean that the pure RSF effect occurs; every model labeled by RSF- 
is subject to both the MSW and RSF conversions. We summarize these models in table $2 \bar{i}$. Each model is further categorized into three groups $\mathrm{A}, \mathrm{B}$ and $\mathrm{C}$ as shown in the table, owing to the expected $\bar{\nu}_{e}$ signal at SK; this greatly simplifies following discussions.

\begin{tabular}{lllll}
\hline Model & $B_{0}(\mathrm{G})$ & Mass hierarchy & $\sin ^{2} 2 \theta_{13}$ & Group \\
\hline MSW-NOR-S & 0 & Normal & $10^{-6}$ & $\mathrm{~A}$ \\
MSW-NOR-L & 0 & Normal & 0.04 & $\mathrm{~A}$ \\
MSW-INV-S & 0 & Inverted & $10^{-6}$ & $\mathrm{~A}$ \\
MSW-INV-L & 0 & Inverted & 0.04 & $\mathrm{~B}$ \\
RSF-NOR-S & $10^{10}$ & Normal & $10^{-6}$ & $\mathrm{~B}$ \\
RSF-NOR-L & $10^{10}$ & Normal & 0.04 & $\mathrm{~B}$ \\
RSF-INV-S & $10^{10}$ & Inverted & $10^{-6}$ & $\mathrm{~A}$ \\
RSF-INV-L & $10^{10}$ & Inverted & 0.04 & $\mathrm{C}$ \\
\hline
\end{tabular}

Table 2: Models considered in this paper, concerning the neutrino properties.

\section{Results of numerical calculations}

\subsection{Conversion probability}

We calculated equation $(\overline{2} \cdot \overline{2}, \bar{s})$ numerically with the models given in section $\overline{\underline{\beta}}$, , and obtained the conversion probabilities for each flavour. Figure 离(a) shows the conversion probabilities of the original $\nu_{\mu}$ as a function of radius for the RSF-NOR-L model; the neutrino energy is $25 \mathrm{MeV}$. A significant amount of $\nu_{\mu}$ changes into $\bar{\nu}_{\mathrm{e}}$, owing mainly to the RSF-H conversion which occurs around $\sim 0.01 R_{\odot}$, and the converted $\bar{\nu}_{\mathrm{e}}$ propagates as the mass eigenstate due to the matter effect, not being disturbed by further resonances, to $\sim 0.1 R_{\odot}$. Around this radius, $\bar{\nu}_{\mathrm{e}}$ starts to mix with other flavour antineutrinos, reducing the probability $P\left(\nu_{\mu} \rightarrow \bar{\nu}_{\mathrm{e}}\right)$. In figure $\bar{\mu}_{-}^{(}(\mathrm{b})$, we show the conversion probabilities of original $\nu_{\mathrm{e}}$ for the RSF-INV-L model. The original $\nu_{\mathrm{e}}$ are transformed into $\bar{\nu}_{\mu, \tau}$ at RSF-H, and they further change to the favoured flavour $\bar{\nu}_{\mathrm{e}}$ at the MSW-H resonance point.

We show in figure the same probabilities as those shown in figure value of $\theta_{13}$ is small ((a) RSF-NOR-S; (b) RSF-INV-S). Figure $\overline{5}_{1}^{\prime}(a)$ indicates that the conversion probabilities from the original $\nu_{\mu}$ are almost the same as those for RSF-NOR-L model; the value of $\theta_{13}$ does not matter in the case of normal mass hierarchy. On the other hand, the behaviours in figure 它(b) are substantially different from those in figure particular, the most easily detected flavour $\bar{\nu}_{\mathrm{e}}$ is not produced from the original $\nu_{\mathrm{e}}$. All these characteristics are consistent with simple qualitative discussions given in [ii].

\subsection{Neutrino signals at the Super-Kamiokande detector}

With the conversion probabilities given in the previous subsection and the original neutrino spectrum by Thompson et al. [1] the Earth. (From this point on, we assume the galactic supernova neutrino burst and take $10 \mathrm{kpc}$ as the distance to the supernova.) Using this flux and the cross section of the relevant neutrino interaction at SK as well as the sensitivity of the detector, we can 


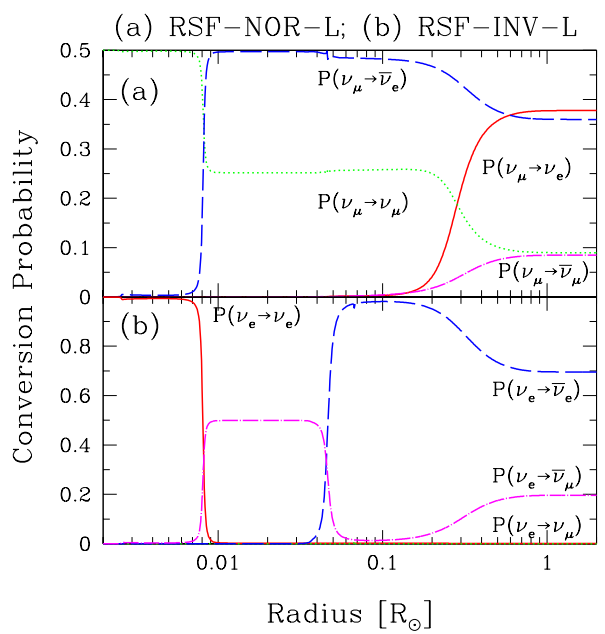

Figure 4: Conversion probabilities as a function of radius for the (a) RSF-NOR-L and (b) RSF-INV-L models. The injected neutrino energy is taken to be $25 \mathrm{MeV}$.

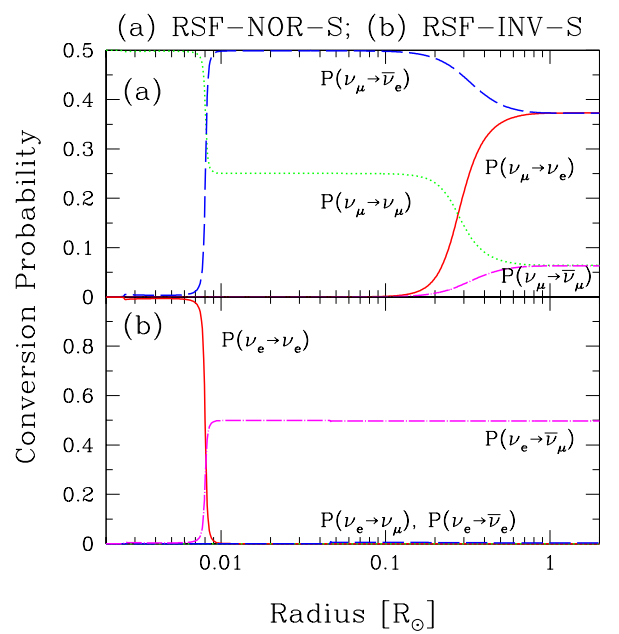

Figure 5: Conversion probabilities as a function of radius for the (a) RSF-NOR-S and (b) RSF-INV-S models. The injected neutrino energy is taken to be $25 \mathrm{MeV}$.

calculate the expected event numbers from a future galactic supernova neutrino burst. In this paper, we adopt the most dominant reaction $\bar{\nu}_{\mathrm{e}} \mathrm{p} \rightarrow \mathrm{e}^{+} \mathrm{n}$ alone; a cross section of the reaction has been calculated in detail [i] $\left.{ }_{1}^{1} \overline{\overline{8}_{1}}\right]$.

Figure $\bar{b}_{(}^{\prime}(\mathrm{a})$ shows the time evolution of the energy-integrated event as a function of time for each group A, B and C, and we show the same in figure $\mathbf{6}_{1}^{6}(\mathrm{~b})$ but using equally spaced bins. From the time evolution of neutrino events, we cannot discern the groups $\mathrm{A}$ and $\mathrm{B}$, because they show almost the same time profile. For group C, however, since the original $\nu_{\mathrm{e}}$ are converted into $\bar{\nu}_{\mathrm{e}}$, the time profile shows a steep neutronization peak, and the event number contained in this peak is expected to be statistically significant, as clearly seen in figure $\sim 180$. If the neutronization peak were actually obtained, it would strongly indicate that the model group $\mathrm{C}$ would be favoured; since the group $\mathrm{C}$ contains only one model, RSFINV-L, a great number of problems concerning the neutrino properties would be solved at the same time. In that case, the neutrino would have the nonzero magnetic moment, the mass hierarchy would be inverted, and the value of $\theta_{13}$ would be large enough for MSW-H to be adiabatic.

The expected event number per unit energy range, which is integrated during the first $0.25 \mathrm{~s}$ after core bounce, is shown in figures $\overline{\underline{6}}_{1}(\mathrm{c})$ and $\overline{\underline{6}}(\mathrm{~d})$, in units of counts/MeV and counts/bin, respectively. From these figures, the model group $\mathrm{C}$ gives the softest spectrum, while $\mathrm{B}$ gives the hardest and $\mathrm{A}$ an intermediate one. In addition to the time evolution of the neutrino events, the number spectrum would provide useful information on the flavour conversion mechanism. Although the available data are restricted in order to avoid uncertainties concerning shock wave propagation, the obtained data would be statistically significant. Using the spectrum, degeneracy between the groups A and B is expected to be broken. 

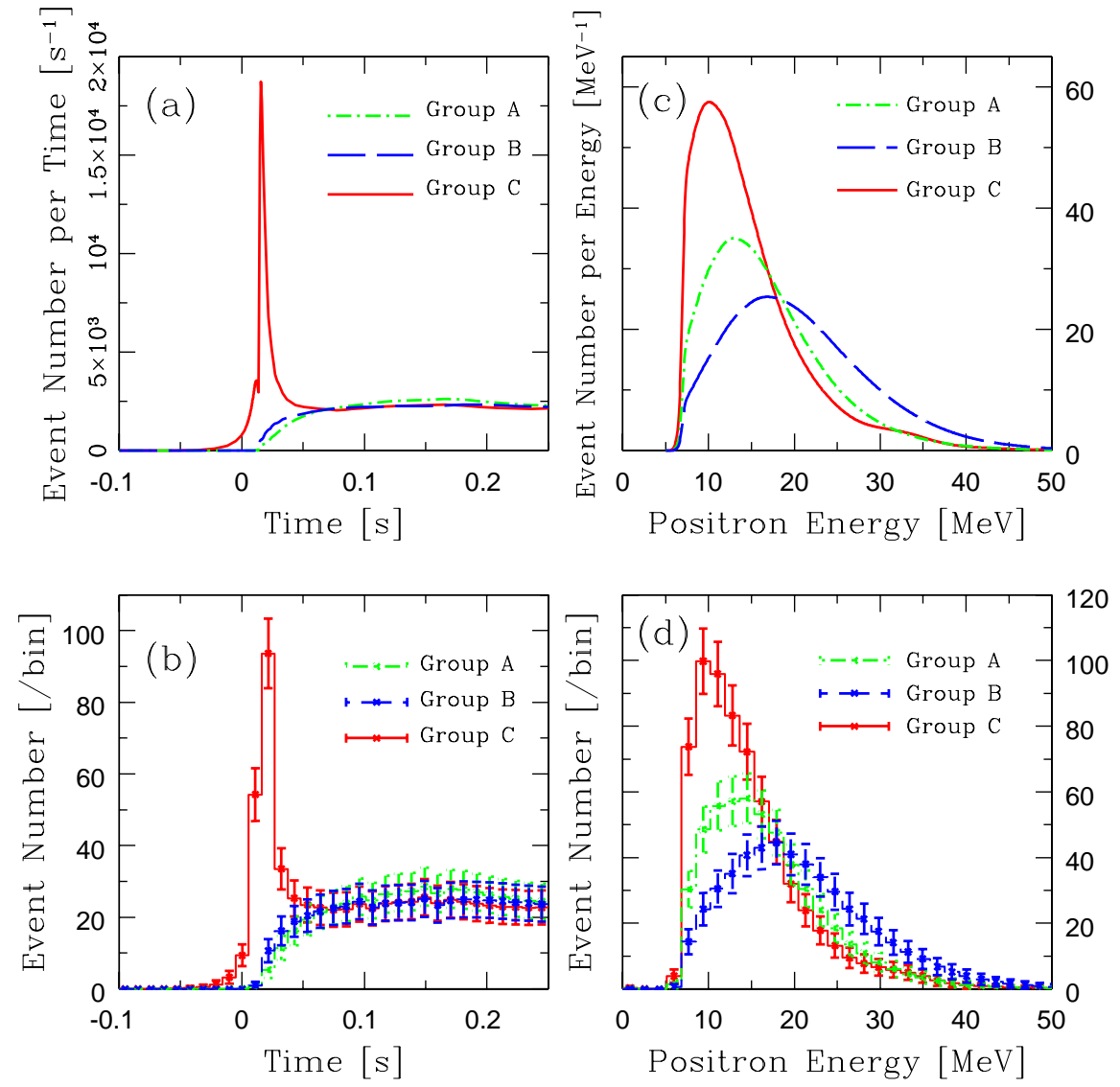

Figure 6: Neutrino signal at the SK detector, which is evaluated for model groups A, B and C. (a) Time evolution of the neutrino signal in units of counts/s; (b) the same as (a), but in units of counts/bin with $1 \sigma$ statistical error bars; (c) number spectrum of positrons for first $0.25 \mathrm{~s}$ after core bounce in units of counts/MeV; (d) the same as (c), but in units of counts/bin with $1 \sigma$ statistical error bars.

\section{Discussion}

The expected neutrino signal at the SK detector has been investigated thus far. However, the mechanism of supernova explosions is quite unclear, since all the reliable numerical simulations have not succeeded in pushing the shock wave to penetrate the entire core. There may be several unknown processes which we have omitted so far, and the original neutrino spectrum as well as its luminosity curve is still controversial. Therefore, we cannot trust the characteristics of the calculation by Thompson et al. [1힘] in detail. Instead, we use rather simple quantities in order to discuss the conversion mechanisms from the neutrino signals; this approach is expected to considerably reduce the dependence on supernova models.

We adopt the following quantities:

$$
R_{\mathrm{SK}}^{\mathrm{E}}=\frac{\text { Number of events for } E_{\mathrm{e}}>25 \mathrm{MeV}}{\text { Number of events for } E_{\mathrm{e}}<15 \mathrm{MeV}}
$$




$$
R_{\mathrm{SK}}^{\mathrm{T}}=\frac{\text { Number of events for } 0<t / \mathrm{ms}<75}{\text { Number of events for } 75<t / \mathrm{ms}<150},
$$

in order to represent the spectral hardness and the peak sharpness of the neutronization burst, respectively. Since the time of the core collapse would never be known with the neutrino signal alone, we take time origin $t=0$ when the first neutrino signal is detected. The places of the model groups $\mathrm{A}, \mathrm{B}$ and $\mathrm{C}$ on the $\left(R_{\mathrm{SK}}^{\mathrm{E}}, R_{\mathrm{SK}}^{\mathrm{T}}\right)$ plane are shown in figure These groups are well separated from each other, and we expect that this particular remains unchanged even if the adopted supernova model is different.

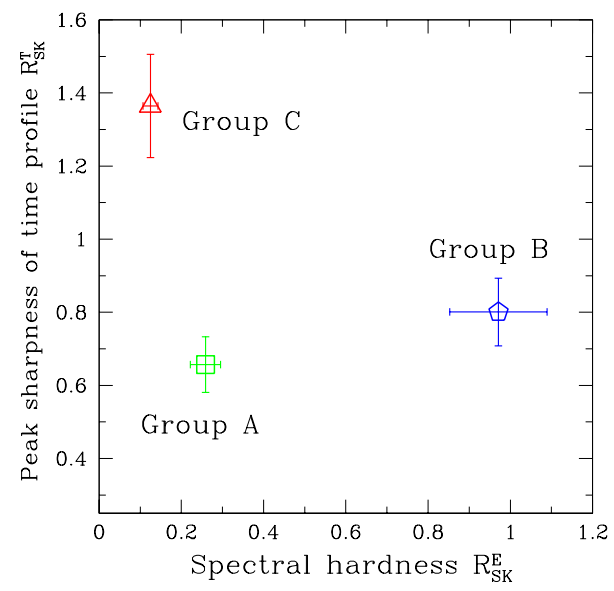

Figure 7: The model groups A, B and C, plotted on the $R_{\mathrm{SK}}^{\mathrm{E}}$ against $R_{\mathrm{SK}}^{\mathrm{T}}$ plane. The error bars include only statistical errors, and are at the $1 \sigma$ level.

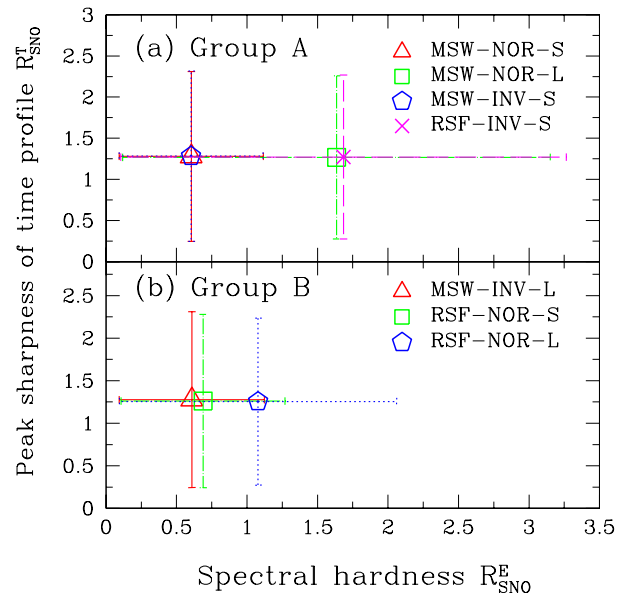

Figure 8: The models in groups A and B, plotted on the $R_{\mathrm{SNO}}^{\mathrm{E}}$ against $R_{\mathrm{SNO}}^{\mathrm{T}}$ plane. The error bars include only statistical errors, and are at the $1 \sigma$ level.

Although the degeneracy problem within each group cannot be solved by the SK observation, which mainly detects $\bar{\nu}_{\mathrm{e}}$, it may be possible if $\nu_{\mathrm{e}}$ could be detected efficiently. SNO is such a detector currently data taking with 1000 tons of heavy water. The supernova $\nu_{\mathrm{e}}$ can be detected via the $\nu_{\mathrm{e}} \mathrm{d} \rightarrow \mathrm{e}^{-}$pp reaction. Although the $\bar{\nu}_{\mathrm{e}}$ are also detected through a similar reaction, $\bar{\nu}_{\mathrm{e}} \mathrm{d} \rightarrow \mathrm{e}^{+} \mathrm{nn}$, these events could be discriminated using the delayed coincidence technique. Thus at SNO, we expect that only the $\nu_{\mathrm{e}}$ signal can be extracted. Figure $\bar{s}$ is the same as figure $\bar{T}_{1}^{-}$for the SNO detector, but is plotted for the models in (a) group A and (b) group B. As shown in this figure, the degeneracy problem could be solved with the $\nu_{\mathrm{e}}$ data in principle, but the current smallness of the SNO detector prevents a statistically significant discussion.

\section{Acknowledgments}

S.A.'s work is supported by a Grant-in-Aid for JSPS Fellows. K.S.'s work is supported in part by a Grant-in-Aid for Scientific Research provided by the Ministry of Education, Culture, Sports, Science and Technology of Japan through Research Grant No S14102004, Grant-in-Aid for Scientific Research on priority Areas No 14079202. 


\section{References}

[1] S. Ando and K. Sato, J. Cosmol. Astropart. Phys. 10 (2003) 001.

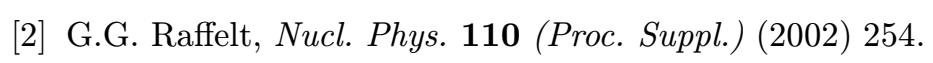

[3] A.S. Dighe and A. Y. Smirnov, Phys. Rev. $\mathbf{D} \mathbf{6 2}(2000) 033007$

K. Takahashi and K. Sato, Prog. Theor. Phys. $109(2003) \overline{19}$.

[4] A. Cisneros, Astrophys. Space Sci. 10 (1970) 87;

K. Fujikawa and R.E. Shrock, 'Phys. Rev. Lett. 45 (1980) 963.

[5] J. Schechter and J.W.F. Valle, 'Phys. Rev. D 24 (1981) $1883 ;$ 'P (erratum).

[6] C.S. Lim and W.J. Marciano, 'Phys. Rev. $\mathbf{D} \mathbf{3 7}(198 \overline{8}) \overline{1} 368$

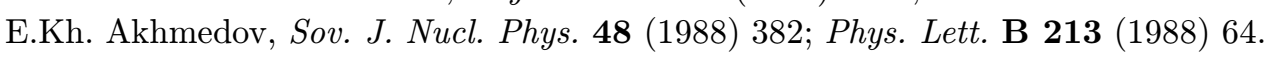

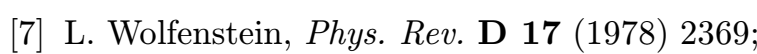
is

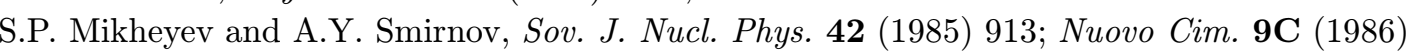

[8] J. Barranco, O.G. Miranda, T.I. Rashba, V.B. Semikoz and J.W.F. Valle, 'Phys. Rev. D66 : -

B.C. Chauhan and J. Pulido, Phys. Rev. D 66 6 20022053006

[9] K. Eguchi et al., 'Phys. Rev. Lett. 90 (2003) 021802;

[10] E. Torrente-Lujan, iJ. High Energy Phys. $04 \overline{(200 \overline{3})} \overline{0} \overline{5} \overline{4}$;

B.C. Chauhan, J. Pulido and E. Torrente-Lujan,

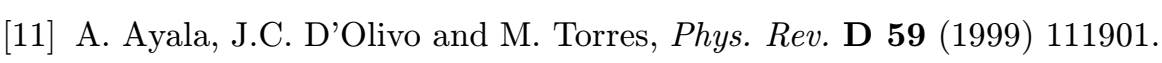

[12] M.B. Voloshin, 'P $\bar{P} \overline{y s}$. Lett. $\mathbf{B} \mathbf{2} \overline{\mathbf{0}} \overline{\mathbf{9}}(\overline{1} \overline{\mathrm{g}} \overline{8} \overline{8}) \overline{360}$;

E.Kh. Akhmedov and Z.G. Berezhiani, Nucl. Phys. B 373 (1992) $479 ;$

E.Kh. Akhmedov, S.T. Petcov and A.Y. Smirnov, Phys.Rev. D 48 199322167 ;

J.T. Peltoniemi, 'Astron. Astrophys. 254 (1992) 1215

H. Athar, J.T. Peltoniemi and A.Y. Smirnov, PThys. Rev. D $5 \overline{1}^{-}(1 \overline{9} \overline{9} \overline{5})^{-} 6 \overline{6} \overline{4}$,

T. Totani and K. Sato,

H. Nunokawa, Y.Z. Qian and G.M. Fuller, Phys. Rev. D 55 (1997) 3265 ;

H. Nunokawa, R. Tomàs and J.W.F. Valle, Ástropart. Phys. 11 (1999) 31 ț.

[13] S. Ando and K. Sato, Phys. Rev. D $67-(2003) 023004$.

[14] S. Ando and K. Sato, 'Phys. Rev. D 68 8 2003 2023003.

[15] T.A. Thompson, A. Burrows and P. Pinto, Astrophys. J. 592 (2003) 4344.

[16] S.E. Woosley and T.A. Weaver, Astrophys. J. Suppl. Ser. 101 (1995) 181.

[17] G. Chanmugam, iAnn. Rev. Astron. Es Astrophys. 411 (1992) 823

[18] P. Vogel and J.F. Beacom, 'P Phys. Rev. D' $\mathbf{6 0}(\overline{1} 9 \overline{9})$ 053003;

A. Strumia and F. Vissani, 'Phys. Lett. B $\mathbf{5 6 4}(2003) 42$.r 